\title{
Epidemiología del cáncer no melanoma en Colombia
}

Epidemiology of non-melanoma skin cancer in Colombia

\section{Javier Cormane ${ }^{1}$, Alcides Rodelo ${ }^{2}$}

1. Médico cirujano, magíster en Gobierno y Políticas Públicas; Director General, Centro Dermatológico Federico Lleras Acosta, E.S.E., Bogotá, D.C., Colombia.

2. Médico, residente de tercer año de Dermatología, Facultad de Medicina, Fundación Universitaria Sanitas, Bogotá, D.C., Colombia.

\section{Resumen}

Ante la ausencia de información epidemiológica sobre el cáncer no melanoma en Colombia, tanto en las estadísticas oficiales como en los registros internacionales, se tomaron y se procesaron los datos institucionales de dos centros de referencia a nivel nacional: el Instituto Nacional de Cancerología y el Centro Dermatológico Federico Lleras Acosta, evidenciándose la magnitud del problema sanitario. Analizada la información se concluye que el cáncer de piel es el tumor maligno más frecuente en nuestro medio, responsable de un poco más del $20 \%$ de los diagnósticos nuevos y, de estos cánceres, el subtipo no melanoma es el más habitual con una participación superior al $83 \%$. La tendencia es de crecimiento en la participación del cáncer no melanoma, en contraposición con el descenso institucional que experimenta el melanoma cutáneo. Integrando las dos fuentes, por año se hacen cerca de 1.230 diagnósticos nuevos de la categoría no melanoma, cerca del $6 \%$ del total del país.

Investigadores del Centro Dermatológico y de la Universidad Nacional de Colombia establecieron las tasas nacionales de cáncer no melanoma, mostrando que para el periodo 2003-2007 pasaron de 19 a 34 casos por 100.000 habitantes al año. La mortalidad del cáncer de piel recae, principalmente, en el subtipo melanoma; en el periodo 2004-2010, en el Instituto Nacional de Cancerología se hizo un registro promedio de veinte fallecidos por melanoma al año.

PALABRAS CLAVE: cáncer de piel, incidencia, mortalidad, Colombia

\section{Summary}

In the absence of epidemiological information on non-melanoma cancer in Colombia, both in official statistics and international registries, institutional data from two reference centers (Instituto Nacional de Cancerología and Centro Dermatológico Federico Lleras Acosta) were taken and processed to show the magnitude of this health problem. After analyzing the information we concluded that skin cancer is the most common malignant tumor in our country, being responsible for more than $20 \%$ of new diagnoses, and the most common non-melanoma cancer subtype, over $83 \%$. The growing trend of non-melanoma cancer is evident as opposed to the institutional decrease of cutaneous melanoma. Integrating the above mentioned sources, about 1,230 new diagnoses of non-melanoma cases per year are made, about $6 \%$ of the national total.

Researchers from the Centro Dermatológico Federico Lleras Acosta and the Universidad Nacional de Colombia established national rates of non-melanoma

\section{Correspondencia:}

Javier Cormane

\section{Email:}

director@dermatologia.gov.co

Recibido: 16 de diciembre de 2013. Aceptado: 15 de enero de 2014.

No se reportan conflictos de interés. 
cancer, showing that for the period 2003-2007 it had increased from 19 to 34 cases per 100,000 inhabitants per year. Skin cancer mortality falls mainly in the melanoma subtype in the period 2004-2010, at the Instituto Nacional de Cancerología, with a mean of 29 deaths per year.

KEY WORDS: skin cancer, incidence, mortality, Colombia

En Colombia no se cuenta con datos estadísticos oficiales sobre las neoplasias cutáneas en general, a pesar de que el cáncer es una enfermedad de interés en salud pública y de prioridad para el país, como lo establece la Ley 1384 de 2010, más conocida como Ley Sandra Ceballos, y los acuerdos vinculantes de la Organización Panamericana de la Salud (OPS) y la Organización Panamericana de la Salud (OMS).

El Sistema de Vigilancia Epidemiológica (Sivigila) en su componente centinela en salud pública, no incluye el reporte obligatorio del cáncer de piel, como sí lo hace para otros cánceres, como los hematológicos, por parte de las unidades primarias generadoras del dato ${ }^{1}$.

Por otra parte, el recién creado Observatorio Nacional de Salud tampoco contempla el seguimiento a las novedades epidemiológicas de los tumores dermatológicos malignos ${ }^{2}$. En el Plan Decenal de Salud Pública, 2012-2021, se dispone como meta número 19, entre las dimensiones prioritarias en 'salud ambiental: hábitat saludable', que al año 2021 estará en implementación el sistema de vigilancia de cáncer de piel asociado a la exposición a radiaciones ultravioleta ${ }^{3}$.

Por otro lado, el Registro Poblacional (sic.) de Cáncer de Cali, cuya información forma parte de los registros mundiales de cáncer publicados por la International Agency for Research on Cancer (IARC), ha publicado en Cancer Incidence in Five Continents las tasas del melanoma cutáneo en Cali, desde 1965 hasta $2007^{4}$; sin embargo, no se cuenta en el país con datos de población que incluyan el cáncer no melanoma, el tumor maligno más frecuente.

Por todo lo anterior, y para revisar los aspectos epidemiológicos del cáncer no melanoma en Colombia, se tomó la información del registro institucional de cáncer del Instituto Nacional de Cancerología y de las investigaciones desarrolladas por el Centro Dermatológico Federico Lleras Acosta, ambas entidades en cumplimiento de sus objetivos sociales, el primero, como centro de referencia para el cáncer en general, y el segundo, para el cáncer cutáneo y en la delegación gubernativa de la vigilancia y control del cáncer de piel ${ }^{5,6}$. Es pertinente anotar que el Instituto Nacional de Cancerología ofrece servicios de mayor complejidad para la atención del cáncer de piel con destino a la asistencia del cáncer no melanoma en estadios avanzados y al melanoma cutáneo; esto genera algunas diferencias en las estadísticas institucionales con respecto al Centro Dermatológico Federico Lleras Acosta, que atiende más condiciones cancerígenas para los primeros estadios del cáncer no melanoma.

\section{Registro institucional del cáncer en el Instituto Nacional de Cancerología}

\section{Morbilidad del cáncer primario en piel, 2004-2010}

En la primera tabla del anuario estadístico del Instituto Nacional de Cancerología se encuentra la distribución de casos nuevos de cáncer por sexo, según su localización primaria. Allí, bajo el código $\mathrm{C} 44$, se identifica el cáncer de piel. En la TABLA 1 se presenta el número de diagnósticos nuevos de cáncer primario en piel, independientemente del sexo, entre los años 2004 y $2010^{7-13}$. Los datos denotan una incidencia institucional relevante para esta enfermedad, con un rango entre los 880 y los 1.020 casos nuevos por año, en el periodo disponible. El cáncer de piel presenta una tendencia al incremento en el número de casos diagnosticados en el Instituto Nacional de Cancerología (Figura 1, LÍNEA PUNTEADA).

En la Tabla 6 del anuario estadístico del Instituto Nacional de Cancerología se encuentran los casos nuevos de cáncer de piel de localización primaria (código C44), según su diagnóstico histológico. Para facilitar la presentación de los datos se agrupan los diagnósticos que aparecen en la publicación con la Clasificación Estadística Internacional de Enfermedades y Problemas Relacionados con la Salud en su décima revisión, en cáncer no melanoma y melanoma cutáneo, y se cuantifica la incidencia institucional por 


\begin{tabular}{llllllll}
\hline Años & 2004 & 2005 & 2006 & 2007 & 2008 & 2009 & 2010 \\
Casos (n) & 906 & 1.011 & 956 & 895 & 880 & 954 & 1.273 \\
\hline
\end{tabular}

TABLA 1. Casos nuevos de cáncer primario en piel, Instituto Nacional de Cancerología, 2004-2010.

Fuente: anuarios estadísticos del Instituto Nacional de Cancerología ${ }^{7-13}$.

Disponibles en: www.cancer.gov.co/contenido/contenido.aspx?catID $=437 \&$ conID $=747$

\begin{tabular}{llllllll}
\hline Años & 2004 & 2005 & 2006 & 2007 & 2008 & 2009 & 2010 \\
Cáncer no melanoma (n) & 758 & 836 & 832 & 777 & 760 & 811 & 1.130 \\
Melanoma cutáneo $(\mathrm{n})$ & 148 & 175 & 124 & 118 & 120 & 143 & 143 \\
\hline
\end{tabular}

TABLA 2. Casos nuevos de cáncer de piel primario, según el subtipo: cáncer no melanoma o melanoma cutáneo, Instituto Nacional de

Fuente: anuarios estadísticos del Instituto Nacional de Cancerología (7-13). Cancerología, 2004-2010

Disponibles en: $w w w$. cancer.gov.co/contenido/contenido.aspx? catID $=437 \&$ conID $=747$

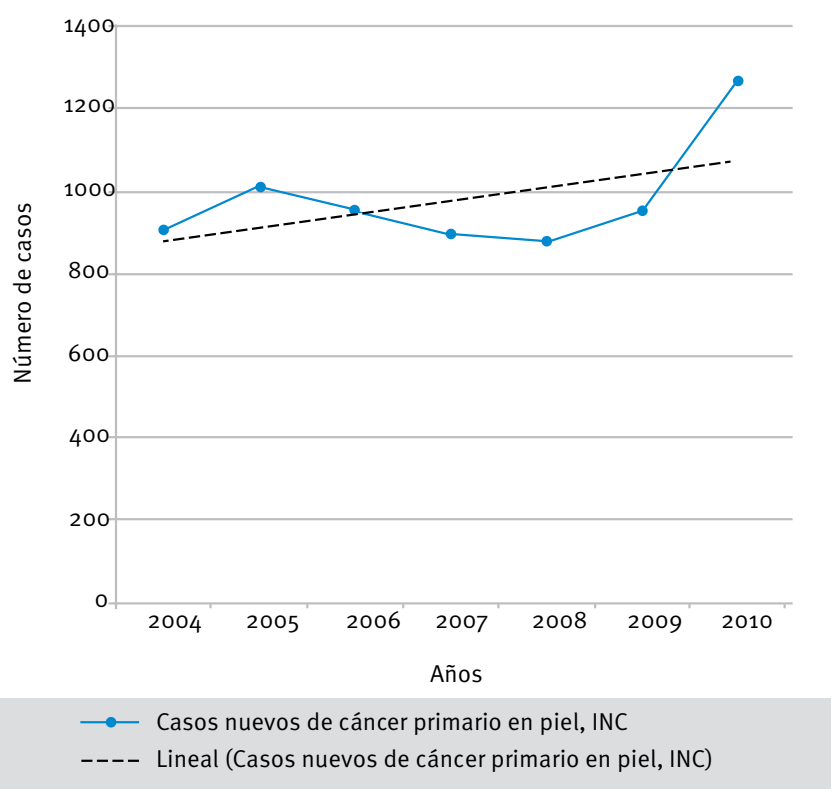

FiguRA 1. Casos nuevos de cáncer primario de piel, Instituto Nacional de Cancerología (INC), 2004-2010 $0^{7-13}$.

cada uno de los dos subtipos de cáncer de piel por año del periodo disponible.

En la TABLA 2 se observa que el cáncer no melanoma predomina sobre el melanoma cutáneo en el Instituto Nacional de Cancerología, en todos los años, con una relación de 6 a 1. En el periodo 2004-2010, el cáncer no melanoma obtuvo una participación, en el total de cánceres de piel, de 83,6 \%, con un acumulado de 5.904 nuevos casos y una media por año de 843 diagnósticos.

La tendencia del cáncer no melanoma, en el periodo 2004-2010, es creciente, como se muestra en la Figura 2, lo que quiere decir que se debe esperar que continúe el incremento de casos nuevos de este

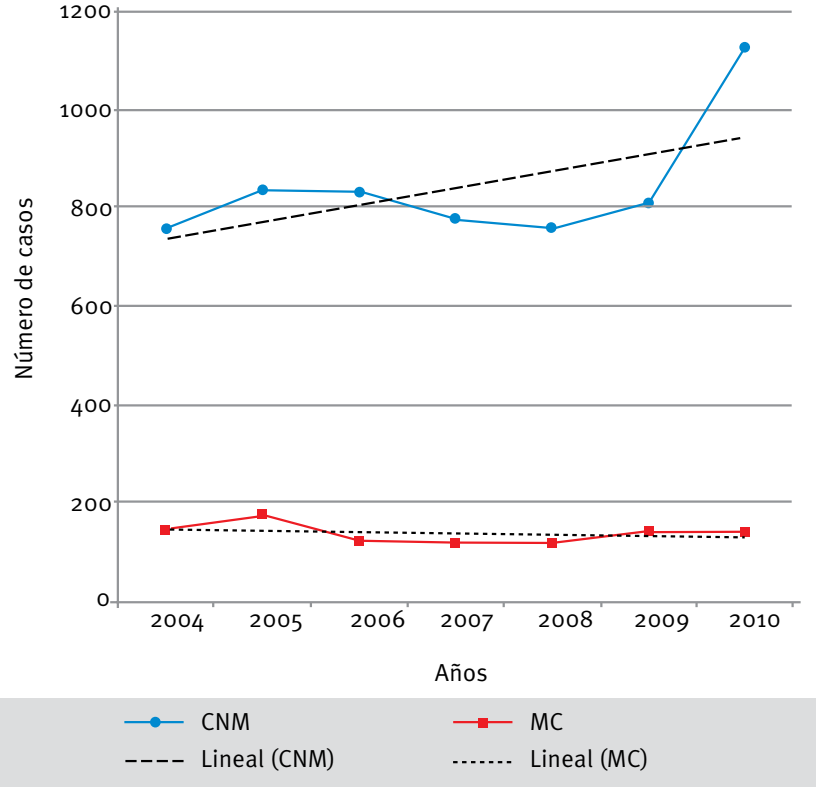

FiguRA 2. Casos nuevos de cáncer primario de piel, según el subtipo: cáncer no melanoma(CNM) o melanoma cutáneo (MC), Instituto Nacional de Cancerología, 2004-2010 ${ }^{7-13}$.

subtipo de cáncer de piel en el Instituto Nacional de Cancerología en los años venideros; por ello, la importancia de la intervención sanitaria de sus factores determinantes.

Por su parte, la incidencia institucional de melanoma cutáneo se mantiene estable, en un rango entre 118 y 175 diagnósticos. En la FIGURA 2, el melanoma cutáneo muestra una tendencia decreciente en el número de casos nuevos en el Instituto, en contraposición a lo que sucede con el cáncer no melanoma.

Con los casos nuevos de cáncer de piel por año, de la Tabla 1 del anuario del Instituto Nacional de Cancerología, y el total de casos diagnosticados en el Instituto 


\begin{tabular}{llllllll}
\hline Años & 2004 & 2005 & 2006 & 2007 & 2008 & 2009 & 2010 \\
Casos (\%) & 15 & 17,1 & 18,3 & 16,9 & 16,2 & 17,6 & 20,2 \\
\hline
\end{tabular}

Fuente: anuarios estadísticos del Instituto Nacional de Cancerología (7-13)

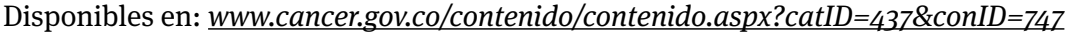

\begin{tabular}{llllllll}
\hline Años & 2004 & 2005 & 2006 & 2007 & 2008 & 2009 & 2010 \\
Casos (n) & 28 & 17 & 28 & 16 & 16 & 18 & 14 \\
\hline
\end{tabular}

Fuente: anuarios estadísticos del Instituto Nacional de Cancerología (7-13)

Disponibles en: $w$ ww.cancer.gov.co/contenido/contenido.aspx? catID $=437 \&$ conID $=747$

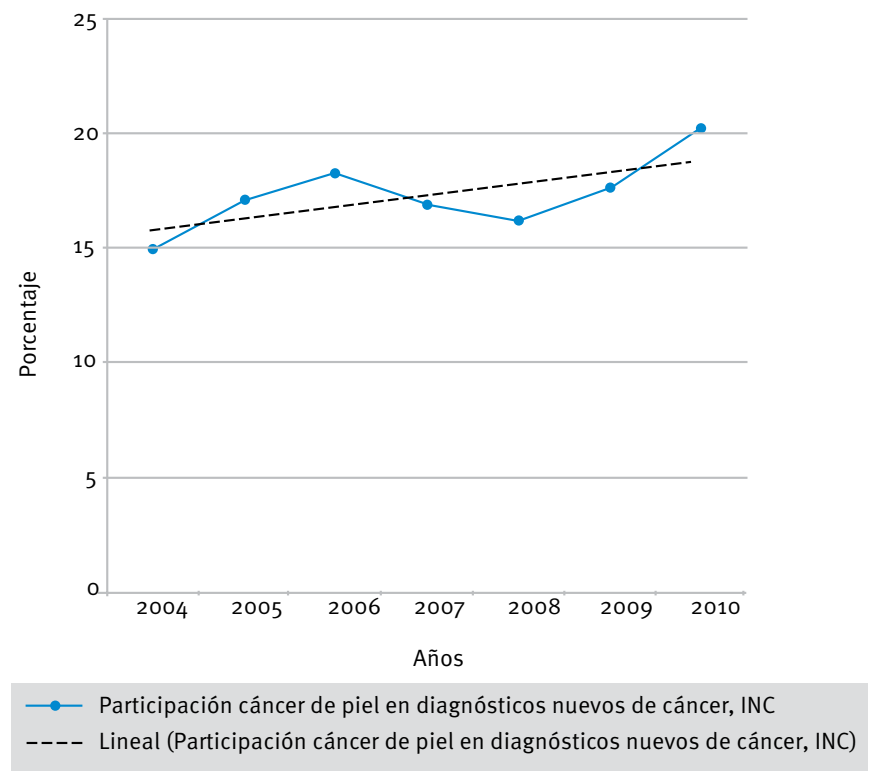

FIGURA 3. Participación de los casos nuevos de cáncer primario de piel del total de diagnósticos nuevos de cáncer, Instituto Nacional de Cancerología (INC), 2004-2010 ${ }^{7-13}$.

de cualquier tipo de cáncer por año, que proviene también de la primera tabla, se obtiene la participación del cáncer de piel en el espectro oncológico del centro de referencia. En la TABLA 3 se observa la participación en el periodo 2004-2010 del cáncer de piel sobre el total de cánceres en el Instituto, en porcentajes, resaltando que en el periodo de análisis nunca estuvo por debajo del $15 \%$ y obtuvo un máximo del 20,2\%; con este último dato se puede decir que la quinta parte de los cánceres diagnosticados en el centro de referencia en el 2010, fueron de piel, siendo de gran peso la participación de esta enfermedad en la morbilidad general oncológica institucional.

Los tres grupos principales de tumores malignos en el anuario estadístico del Instituto Nacional de Cancerología del 2010, en orden descendente, siguen siendo: piel $(20,2 \%)$, sistema digestivo $(15,9 \%)$ y aparato genital femenino (15,4\%); sin embargo, los dos últimos comienzan a disminuir su incidencia institucional y, por ende, su participación en el periodo 2004-2010, lo cual podría estar relacionado con el impacto de los planes, programas y procedimientos que ha establecido el gobierno nacional central para estos tipos de cáncer. La tendencia de la participación, en porcentaje, del cáncer de piel sobre el total de cánceres diagnosticados en el Instituto es de crecimiento (FIGURA 3, LÍNEA PUNTUADA NEGRA).

\section{Mortalidad por cáncer primario de piel, 2004-2010}

En las tablas 26 y 27 del anuario del Instituto Nacional de Cancerología se presenta la información de la mortalidad institucional del melanoma cutáneo, tanto hospitalaria como en el domicilio del enfermo, según causa básica de defunción y sexo. El Instituto no publica la mortalidad específica del cáncer no melanoma, sino que la incluye en el resto de tumores malignos, por lo que no se puede hacer un análisis específico de este subtipo de cáncer de piel.

Para fines prácticos, en la TABLA 4 se presenta el total de fallecidos por melanoma cutáneo del 2004 al 2010, independiente del sexo. Excepto en los años 2004 y 2006, cuando se presentaron 28 muertes en cada uno, la mortalidad institucional por melanoma cutáneo es estable, con una tendencia franca a la baja (FIGURA 4, LÍNEA PUNTEADA NEGRA). Es decir, la propensión es a tener menos muertes por melanoma cutáneo en los años subsiguientes. El promedio de mortalidad institucional, en el periodo objeto de revisión, es de 20 , hospitalaria o en el domicilio. 


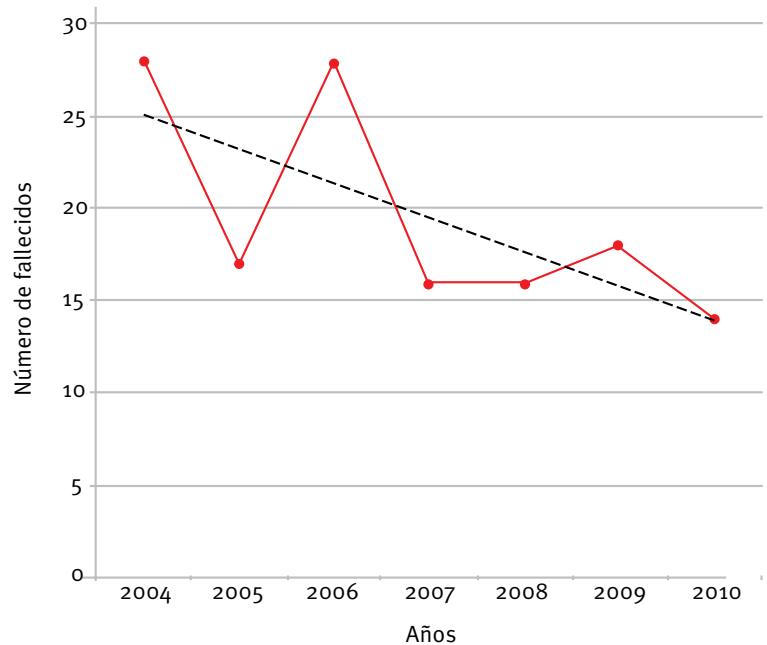

$\longrightarrow$ Casos de muerte por MC, INC

---- Lineal (Casos de muerte por MC, INC)

FIGURA 4. Defunciones institucionales por melanoma cutáneo (MC), tanto hospitalarias como en el domicilio, Instituto Nacional de Cancerología (INC), 2004-2010 $0^{7-13}$.

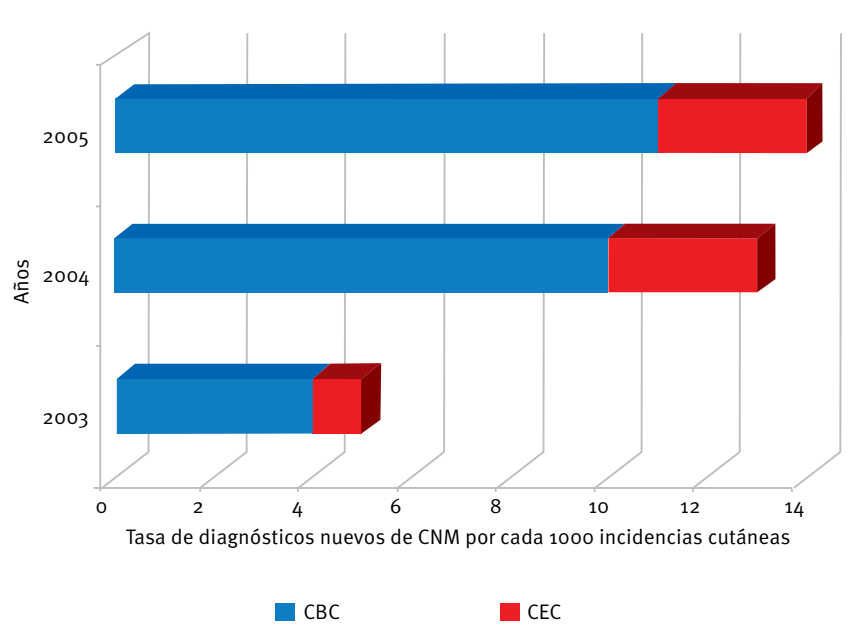

FiguRA 5. Tasa de diagnósticos nuevos de cáncer no melanoma por 1.000 incidencias cutáneas, según variantes histológicas: cáncer basocelular (CBC) y cáncer escamocelular (CEC), Centro Dermatológico Federico Lleras Acosta, 2003-200 $5^{14}$. Reproducido con autorización de: Revista de Salud Pública (Bogotá).

\begin{tabular}{ccccccc}
\hline \hline \multirow{2}{*}{ Años } & $\begin{array}{c}\text { Diagnósticos } \\
\text { confirmados }\end{array}$ & \multicolumn{2}{c}{ Cáncer basocelular } & \multicolumn{2}{c}{ Cáncer escamocelular } & \multicolumn{2}{c}{ Cáncer no } \\
& $\mathrm{n}$ & Tasa & $\mathrm{N}$ & Tasa & melanoma n \\
\hline 2003 & 55.913 & 224 & 4 & 75 & 1 & 299 \\
2004 & 54.323 & 527 & 10 & 167 & 3 & 694 \\
2005 & 58.423 & 661 & 11 & 191 & 3 & 852 \\
\hline \hline
\end{tabular}

TABLA 5. Tasas de cáncer basocelular y cáncer escamocelular por 1.000 diagnósticos confirmados de primera vez por año, Centro Dermatológico Federico Lleras Acosta, 2003-2005..

Reproducido y modificado con autorización de: Nova J, Sánchez G, Porras L. Skin cancer: A Colombian reference centre’s epidemiological profile, 2003-2005. Rev Salud Pública (Bogotá). 2007;9:595-601.

\section{Investigaciones desarrolladas} y publicadas por el Centro

\section{Dermatológico Federico Lleras}

\section{Acosta}

\section{Tasas de casos nuevos de cáncer no melanoma, 2003-2005}

El Centro Dermatológico Federico Lleras Acosta llevó a cabo un estudio descriptivo basado en los registros del sistema de información integral de la institución de los años 2003 al 2005, con el fin de establecer el número de diagnósticos nuevos correspondientes a enfermedad tumoral maligna en piel ${ }^{14}$. En la TABLA 5 se despliegan los datos del estudio para las variantes histopatológicas basocelular y escamocelular, así como el total de diagnósticos nuevos de cáncer no melanoma. En el periodo del estudio, el total de cáncer no melanoma fue de 1.845, excluidos los linfomas y otros tumores malignos indiferenciados, y el de melanoma cutáneo fue de apenas 137, para una participación del cáncer no melanoma de 92,6\% del total de cánceres de piel en el Centro Dermatológico Federico Lleras Acosta. A pesar de la significancia en el número de casos, el cáncer de piel no forma parte de las primeros diez diagnósticos del perfil epidemiológico del Centro en el periodo del estudio.

Entre el 2003 y el 2005, se evidenció un incremento de la incidencia institucional del cáncer no melanoma, pasando de 299 a 852 casos, con un crecimiento cercano al $65 \%$ y un promedio al año de 615 casos en el Centro Dermatológico Federico Lleras Acosta. En la variante basocelular se pasó de una tasa de 4 a una de 11 por 1.000 diagnósticos confirmados nuevos, y en el cáncer 


\begin{tabular}{cccccc}
\hline & 2003 & 2004 & 2005 & 2006 & 2007 \\
\hline Población mayor de 29 años & $17^{\prime}$ '529.878 & $17^{\prime}$ '915.360 & $18^{\prime} 314.585$ & $18^{\prime} 698.971$ & $19^{\prime} 110.824$ \\
Casos nuevos & 4.001 & 4.796 & 6.426 & 6.949 & 7.805 \\
Incidencia por 100.000 & 23 & 27 & 35 & 37 & 41 \\
\hline
\end{tabular}

TABLA 6. Incidencia de cáncer de piel por 100.00o habitantes en Colombia, estandarizada por edad, 2003-2007.

Reproducido con autorización de: Sánchez G, De la Hoz F, Castañeda C, Nova J. Incidencia de cáncer de piel en Colombia, años 2003-2007. Piel. 2011;26:171-7.

escamocelular, de una tasa de 1 a una de 3 por 1.000 diagnósticos confirmados nuevos, triplicándose ambas variantes del cáncer no melanoma, en el lapso 20032005 (FIGURA 5). El cáncer basocelular es la variante de mayor participación en el cáncer no melanoma, cercana a $75 \%$; el resto corresponde a los casos con diagnóstico de cáncer escamocelular.

\section{Incidencia del cáncer de piel en Colombia, 2003-2007}

El Centro Dermatológico Federico Lleras Acosta y la Universidad Nacional de Colombia llevaron a cabo un estudio ecológico ${ }^{15}$ a partir de tres fuentes nacionales de información consolidada. En la TABLA 6 se presentan en forma consolidada, los resultados obtenidos con la metodología aplicada por los investigadores. Se observa que la incidencia de cáncer de piel en Colombia pasó de 23 casos por 100.000 habitantes en 2003 a 41 casos por 100.000 en 2007 , con una alarmante tendencia ascendente, cuando en un periodo de cinco años se han doblado las tasas de incidencia, y una proyección esperada para el año 2020 de aproximadamente 102 casos por 100.000 habitantes.

El incremento se produjo, fundamentalmente, a expensas del número de casos nuevos de cáncer no melanoma (FIGURA 6) para el que se calcularon tasas de 19 por 100.000 en 2003, incrementándose a 34 por 100.000 en 2007 , con tendencia a continuar en aumento en la población colombiana. Sin embargo, en los casos de melanoma se observa un pequeño incremento, pasando de 4 casos por 100.000 habitantes en 2003 a 6 por 100.000 en 2007.

\section{Discusión}

El cáncer de piel es la enfermedad tumoral maligna de mayor frecuencia en Estados Unidos, Australia y España, preponderantemente en la categoría de cáncer

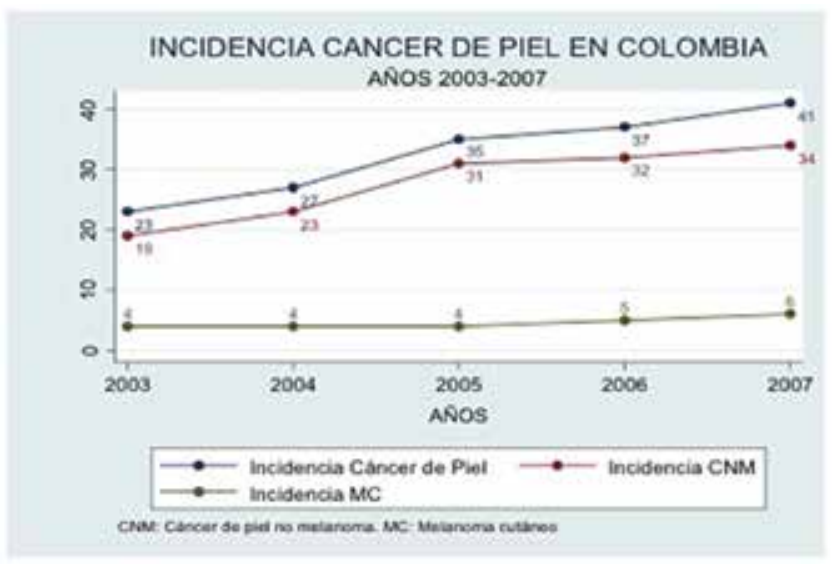

FIGURA 6. Incidencia de cáncer de piel por cada 100.000 habitantes en Colombia, según el subtipo: cáncer no melanoma o melanoma cutáneo, 2003-2007 $7^{15}$.

Reproducido con autorización de: Revista Piel (Barcelona).

no melanoma ${ }^{16-18}$, lo cual genera una preocupación epidemiológica y un accionar de lo público para su control, variables que también se aplican en Colombia. Sin embargo, estos países referenciados han desarrollado sistemas de información, oportunos y confiables, que permiten no solo dimensionar y vigilar la situación sanitaria, sino verificar el impacto de las políticas saludables implantadas ${ }^{19}$, contrario a la deficiencia actual que tenemos en el país, que debe estar superada a más tardar en ocho años.

La información institucional revisada ratifica que de todo el espectro oncológico, el cáncer de piel es el de mayor presencia en nuestro medio y, de este tipo, la categoría de cáncer no melanoma es la más frecuente. No se encuentran registros o estudios específicos de mortalidad por cáncer no melanoma en Colombia, pero se puede resaltar que, en investigaciones en el área oceánica continental norteamericana, las tasas de mortalidad reportadas para el no melanoma son de 0,9 por 
100.00o personas al año ${ }^{20,21}$. El cáncer no melanoma se convierte en centro de la intervención de salud pública y de políticas públicas en tumores dermatológicos malignos, primero, por su presencia predominante en el total de cánceres de piel, y segundo, por la sensibilidad del factor determinante principal -la radiación ultravioleta- a las acciones de prevención.

El gran reto, en adelante, es implantar estrategias efectivas para desacelerar la tendencia creciente en la incidencia del cáncer de piel en Colombia, que amenaza con igualarse a la española, que es de 97 por cada 100.000 habitantes por año ${ }^{18}$. El Plan Decenal para el Control del Cáncer es un primer acercamiento gubernamental ${ }^{22}$.

\section{Referencias}

1. República de Colombia, Ministerio de la Protección Social. Decreto número 3518 de 2006 por el cual se crea y reglamenta el Sistema de Vigilancia en Salud Pública y se dictan otras disposiciones. Bogotá: Ministerio de la Protección Social; 2006

2. República de Colombia, Congreso de Colombia. Ley 1438 de 2011, segunda reforma al Sistema General de Seguridad en Salud, artículos $8^{\circ}$ y subsiguientes, creación del Observatorio Nacional de Salud. Bogotá: Congreso de Colombia; 2011.

3. República de Colombia, Ministerio de Salud y Protección Social. Resolución 1841 de 2013 por la cual se adopta el Plan Decenal de Salud Pública, 2012-2021. Bogotá: Ministerio de Salud y Protección Social; 2013

4. International Agency for Research on Cancer. Cancer incidence in five continents. IARC Sci Publ. 2008;9:118.

5. República de Colombia, Ministerio de Salud. Decreto número 1177 de 1999 por el cual se reestructura el Instituto Nacional de Cancerología, Empresa Social del Estado. Bogotá: Ministerio de Salud; 1999

6. República de Colombia, Ministerio de Salud. Decreto número 1257 de 1994 por el cual se transforma la Unidad Administrativa Especial Centro Dermatológico Federico Lleras Acosta en Empresa Social del Estado del Orden Nacional. Bogotá: Ministerio de Salud; 1994.

7. Instituto Nacional de Cancerología. Anuario Estadístico 2004. ed. Bogotá: MediLegis; 2005. p. 1-84.
8. Instituto Nacional de Cancerología. Anuario Estadístico 2005. Bogotá: MediLegis; 2006. p. 1-98.

9. Instituto Nacional de Cancerología. Anuario Estadístico 2006. Bogotá: MediLegis; 2007. p. 1-96.

10. Instituto Nacional de Cancerología. Anuario Estadístico 2007. Bogotá: MediLegis; 2008. p. 1-96.

11. Instituto Nacional de Cancerología. Anuario Estadístico 2008. Bogotá: MediLegis; 2009. p. 1-99.

12. Instituto Nacional de Cancerología. Anuario Estadístico 2009. Bogotá: Buenos y Creativos; 2011. p. 1-98.

13. Instituto Nacional de Cancerología. Anuario Estadístico 2010. Bogotá: Buenos y Creativos; 2012. p. 1-98.

14. Nova J, Sánchez G, Porras L. Skin cancer: A Colombian reference centre's epidemiological profile, 2003-2005. Rev Salud Pública (Bogotá). 2007;9:595-601.

15. Sánchez G, De la Hoz F, Castañeda C, Nova J. Incidencia de cáncer de piel en Colombia, años 2003-2007. Piel. 2011;26:171-7.

16. Rogers HW, Weinstock MA, Harris AR, Hinckley MR, Feldman $\mathrm{SR}$, Fleischer $\mathrm{AB}$, et al. Incidence estimate of nonmelanoma skin cancer in the United States, 2006. Arch Dermatol. 2010;146:283-7.

17. Staples MP, Elwood M, Burton RC, Williams JL, Marks R, Giles GG. Non-melanoma skin cancer in Australia: The 2002 national survey and trends since 1985. Med J Aust. 2006;184:6-10.

18. Lomas A, Leonardi-Bee J, Bath-Hextall F. A systematic review of worldwide incidence of nonmelanoma skin cancer. Br J Dermatol. 2012;166:1069-80.

19. Cormane J. Análisis de la política pública en prevención del cáncer de piel en Colombia (tesis). Bogotá: Universidad Externado de Colombia; 2013.

20. Weinstock MA, Bogaars HA, Ashley M, Litle V, Bilodeau E, Kimmel S. Nonmelanoma skin cancer mortality. A population-based study. Arch Dermatol. 1991;127:1194-7.

21. Lewis KG, Weinstock MA. Nonmelanoma skin cancer mortality (1988-200o): The Rhode Island follow-back study. Arch Dermatol. 2004;140:837-42.

22. República de Colombia, Ministerio de Salud y Protección Social. Resolución 1383 de 2013 por la cual se adopta el Plan Decenal par el Control del Cáncer en Colombia, 2012-2021. Bogotá: Ministerio de Salud y Protección Social; 2013 